\title{
Deterministic Nucleation of InP on Metal Foils with the Thin-Film Vapor-Liquid-Solid Growth Mode
}

\author{
Rehan Kapadia, ${ }^{\dagger, \ddagger}$ Zhibin Yu, ${ }^{\dagger, \ddagger}$ Mark Hettick, ${ }^{\dagger, \ddagger}$ Jingsan Xu, ${ }^{\dagger, \ddagger}$ Maxwell S. Zheng, ${ }^{\dagger, \ddagger}$ Cheng-Ying Chen, ${ }^{\dagger, \ddagger}$
} Arunima D. Balan, ${ }^{\dagger, \dagger}$ Daryl C. Chrzan, ${ }^{\dagger, \dagger}$ and Ali Javey ${ }^{*},+\frac{\dagger}{\dagger}$

${ }^{\dagger}$ Electrical Engineering and Computer Sciences, University of California, Berkeley, California 94720, United States

${ }^{\ddagger}$ Materials Sciences Division, Lawrence Berkeley National Laboratory, Berkeley, California 94720, United States

Supporting Information
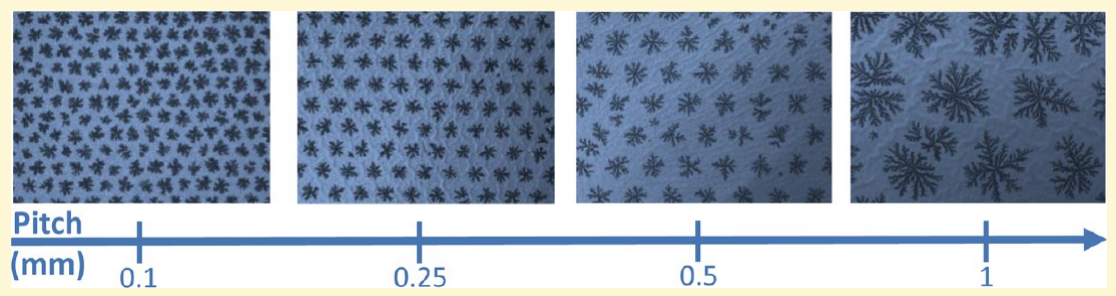

ABSTRACT: A method for growth of ultralarge grain $(>100 \mu \mathrm{m})$ semiconductor thin-films on nonepitaxial substrates was developed via the thin-film vapor-liquid-solid growth mode. The resulting polycrystalline films exhibit similar optoelectronic quality as their single-crystal counterparts. Here, deterministic control of nucleation sites is presented by substrate engineering, enabling user-tuned internuclei spacing of up to $\sim 1 \mathrm{~mm}$. Besides examining the theory associated with the nucleation process, this work presents an important advance toward controlled growth of high quality semiconductor thin films with unprecedented grain sizes on nonepitaxial substrates.

\section{INTRODUCTION}

Photovoltaic devices fabricated from III-V semiconductors offer the highest efficiencies of all classes of materials available, ${ }^{1,2}$ a direct result of the high external luminescence efficiencies of III-V semiconductors. ${ }^{3}$ However, until recently, growth of high quality III-V semiconductors has required expensive epitaxial substrates and metal-organic chemical vapor deposition (MOCVD) processes, ${ }^{4,5}$ offering significant scaling challenges, and relegating high-efficiency III-V devices to niche applications. Recently, we demonstrated growth of poly crystalline InP thin films with grain sizes $>100 \mu \mathrm{m}$ on nonepitaxial substrates using the thin-film vapor-liquid-solid (TF-VLS) growth mode. ${ }^{6}$ TF-VLS growth occurs by passing the phosphorus precursor gas over an In film, which has been capped with $\mathrm{SiO}_{x}$. Phosphorus diffuses through the $\mathrm{SiO}_{x}$ cap at the growth temperatures, causing supersaturation of the liquid In and inducing precipitation of solid InP. As shown schematically in Figure 1 and reported previously, ${ }^{6}$ the process enables the transformation of an entire In film into InP. The mechanisms that enable TF-VLS growth are (i) the inhibition of In dewetting by the template, comprised of the Mo substrate and $\mathrm{SiO}_{x}$ capping layer and (ii) the reduction of incident phosphorus flux by the $\mathrm{SiO}_{x}$ capping layer, enabling lower nucleation densities.

While similar in concept to the vapor-liquid-solid (VLS) growth mode utilized for nanowire growth, ${ }^{7-11}$ the TF-VLS mode enables growth of ultralarge grain continuous thin films, a morphology previously unattainable via the VLS method. Unlike traditional vapor phase growth of polycrystalline materials, ${ }^{12-14}$ TF-VLS growth decouples the lateral nucleation density and film thickness for continuous polycrystalline film growth, enabling lateral grain sizes orders of magnitude larger than film thickness. ${ }^{6}$ Due to the large grain sizes, TF-VLS grown polycrystalline InP exhibited optically measured $V_{\mathrm{oc}}$ (quasi-Fermi level splitting) $\sim 95 \%$ of single crystalline InP. The near single crystal performance of TF-VLS polycrystalline films suggest that this method could play a key role in future thin film optoelectronic devices.

Due to the extreme sensitivity of optoelectronic device quality to defects such as grain boundaries ${ }^{15,16}$ and interfaces, ${ }^{17}$ developing a method for deterministic control of nucleation, in polycrystalline films, is of significant interest for device applications. Previously, we showed that nucleation density in TF-VLS grown films could be controlled by manipulating the phosphorus flux; the nuclei positions, however, were random. Here, we present a general scheme for engineering nucleation in the TF-VLS mode. Specifically, a patterned nucleation promoter (evaporated $\mathrm{MoO}_{x}$ ) is utilized to control the position and density of InP nuclei on Mo foil substrates. Furthermore, a simple model showing quantitative agreement with experiment is presented, leading to a set of design rules for nucleation engineering in TF-VLS grown materials.

\footnotetext{
Received: October 15, 2013

Revised: December 11, 2013

Published: January 17, 2014
} 

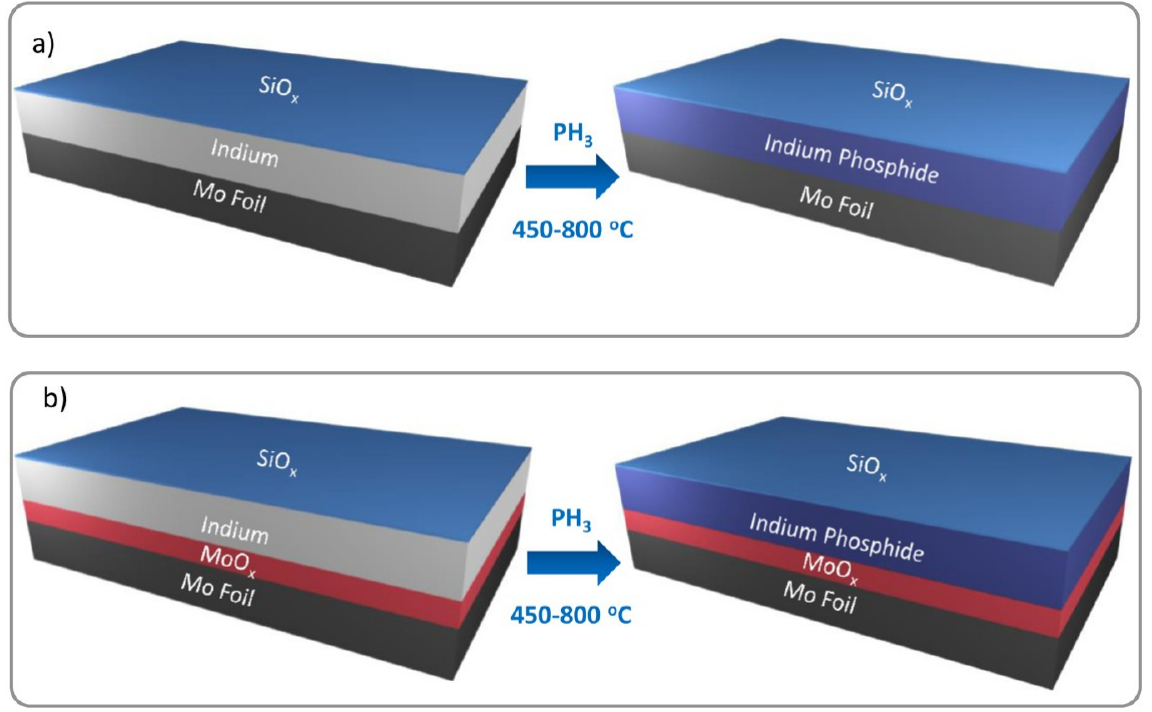

Figure 1. Schematic of TF-VLS process for InP growth on (a) bare Mo foil and (b) Mo foil coated with a 50 nm-thick $\mathrm{MoO}_{x}$ nucleation promoter layer.
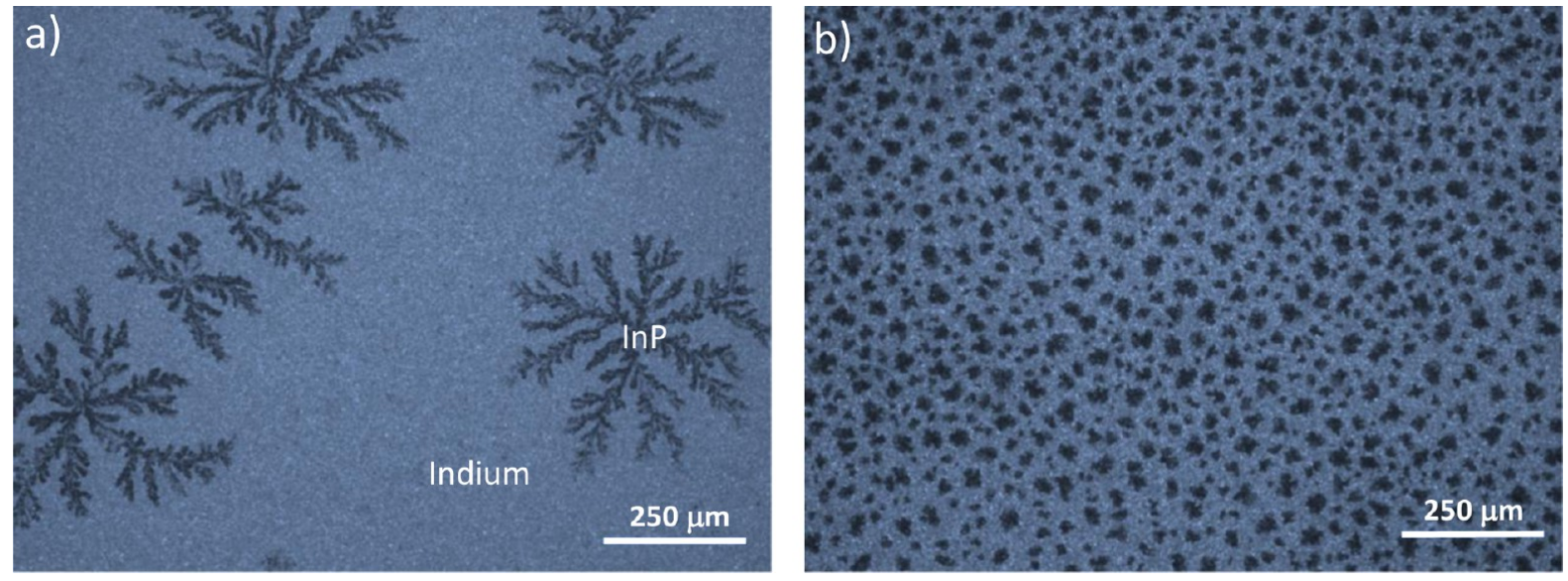

Figure 2. Substrate effects on nucleation density. False color optical microscope images of InP nucleation and growth on (a) a bare Mo foil substrate and (b) a Mo/ $\mathrm{MoO}_{x}$ substrate. Here, the InP films are partially grown by limiting the growth time to clearly depict the nucleation events.

\section{EXPERIMENTAL SECTION}

The first challenge in designing a process for nucleation engineering is finding two materials, a substrate and nucleation promoter, such that the promoter displays nucleation densities orders of magnitude higher than the substrate. To determine the nucleation density differential between two materials, partial growth of $\operatorname{InP}$ films is carried out on both materials under the same conditions. Here, we used Mo foils as the growth substrate and evaporated $\mathrm{MoOx}$ thin films as the nucleation promoter layer. The TF-VLS process was explored on Mo foils $(25 \mu \mathrm{m}, 99.99 \%$, Alfa Aesar) with (Figure $1 \mathrm{~b})$ and without (Figure 1a) a thin $(50 \mathrm{~nm})$ evaporated $\mathrm{MoO}_{x}$ layer. The $\mathrm{MoO}_{x}$ layers were deposited via thermal evaporation utilizing an $\mathrm{Al}_{2} \mathrm{O}_{3}$ coated tungsten evaporation boat and $\mathrm{MoO}_{3}$ powder (99.999\%, Alfa Aesar) as the source at a rate of $\sim 1 \AA / \mathrm{s}$. Mo foils were first degreased with a single set of consecutive $30 \mathrm{~s}$ rinses in acetone, isopropyl alcohol, deionized water, and hydrochloric acid at room temperature. Next, a $1.5 \mu \mathrm{m}$ thick indium film (99.999\%) and a $50 \mathrm{~nm} \mathrm{SiO}{ }_{x}\left(\mathrm{SiO}_{2}\right.$ pellets 99.99\%, Kurt J. Lesker) capping layer were deposited via consecutive e-beam evaporation steps without breaking vacuum. The evaporation rates were $60 \AA / \mathrm{s}$ and $2 \AA / \mathrm{s}$, respectively. Phosphorization of the stacks $\left(\mathrm{Mo} / \mathrm{In} / \mathrm{SiO}_{x}\right.$ and $\left.\mathrm{Mo} / \mathrm{MoO}_{x} / \mathrm{In} / \mathrm{SiO}_{x}\right)$ was carried out in a tube furnace.

For the TF-VLS growth process, the InP films can be quenched in the nucleation, growth, or completion stages by varying the growth time. ${ }^{6}$ Here, InP films are partially grown and quenched before separate InP crystals coalesce. As a result, discrete nucleation sites can be clearly observed, enabling analysis of nucleation density, which dictates the final grain sizes for the fully grown films. The growth was carried out by first heating the substrates in a 1 Torr $\mathrm{H}_{2}$ ambient to $525{ }^{\circ} \mathrm{C}$ followed by phosphorization in a 60 Torr $10 \% \mathrm{PH}_{3}$ ambient for $70 \mathrm{~s}$. The resulting nucleation density is measured by counting the number of independent InP crystals by optical imaging.

\section{RESULTS}

Figure 2 shows optical images of two samples grown under the same conditions, one on a bare Mo substrate (Figure 2a) and the other on a $\mathrm{Mo} / \mathrm{MoO}_{x}$ substrate (Figure $2 \mathrm{~b}$ ). It can clearly be seen that the nucleation density on $\mathrm{MoO}_{x}$ (Figure $2 \mathrm{~b}$ ) is much greater than on bare Mo foil (Figure 2a), suggesting evaporated $\mathrm{MoO}_{x}$ may serve the role of a nucleation promoter for InP growth. In order to confirm that the growing islands are InP, X-ray diffraction and steady-state photoluminescence characterization were performed on the quenched islands (Supporting Information Figure S1). The results indicate that the islands shown are InP. A quantitative nucleation density analysis reveals that the $\mathrm{InP}$ nucleation density, $N$, on the bare Mo substrate is $\sim 450 \mathrm{~cm}^{-2}$, while the nucleation density on the 

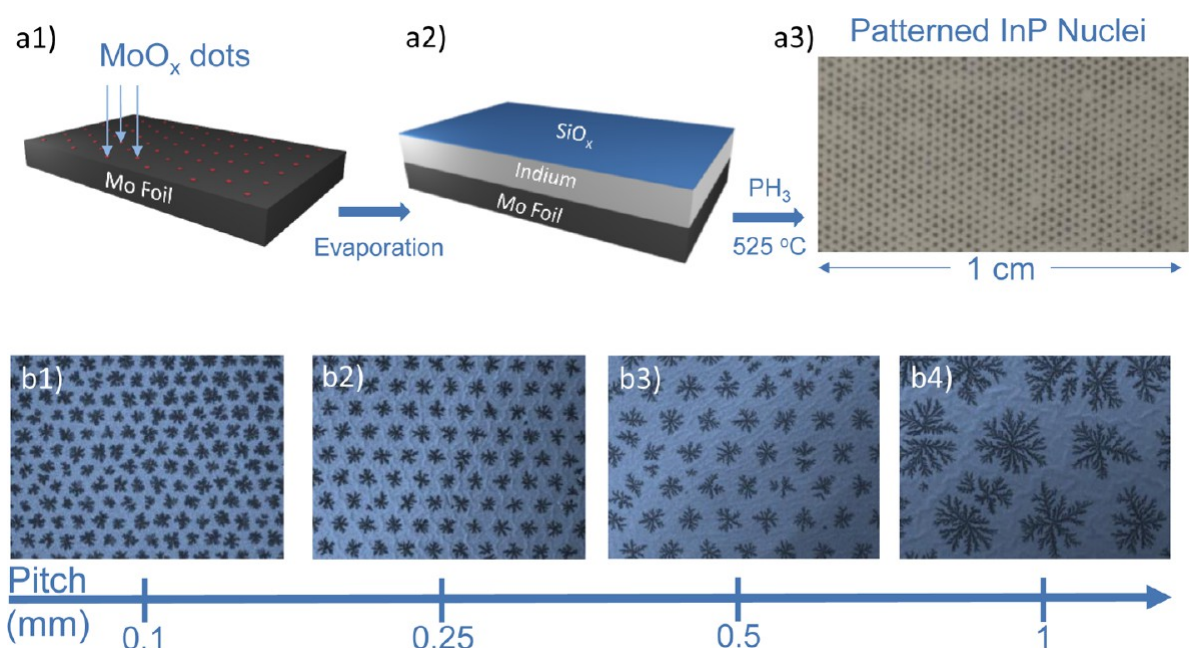

Figure 3. Patterned nucleation. (a1) Schematic of a Mo foil with a hexagonal $\mathrm{MoO}_{x}$ dots as the nucleation sites. Center-to-center pitch, $s$, between the $\mathrm{MoO}_{x}$ pattern is varied between $0.1 \mathrm{~mm}$ to $1 \mathrm{~mm}$. (a2) $\mathrm{In} / \mathrm{SiO}_{x}$ stacks are subsequently evaporated on the patterned $\mathrm{Mo} / \mathrm{MoO}$ substrate. (a3) An optical image of $\sim 1 \mathrm{~cm} \times 0.5 \mathrm{~cm}$ substrate with $0.25 \mathrm{~mm} \mathrm{MoO}$ pitch after partial InP growth, clearly demonstrating large area control over nuclei position. (b1-4) Optical images of partially grown $\mathrm{InP}$ on patterned $\mathrm{Mo} / \mathrm{MoO}_{x}$ substrates with $\mathrm{MoO}_{x}$ pitch of $0.1 \mathrm{~mm}$ (b1), $0.25 \mathrm{~mm}$ (b2), $0.5 \mathrm{~mm}$ (b3), and $1 \mathrm{~mm}(\mathrm{~b} 4)$.
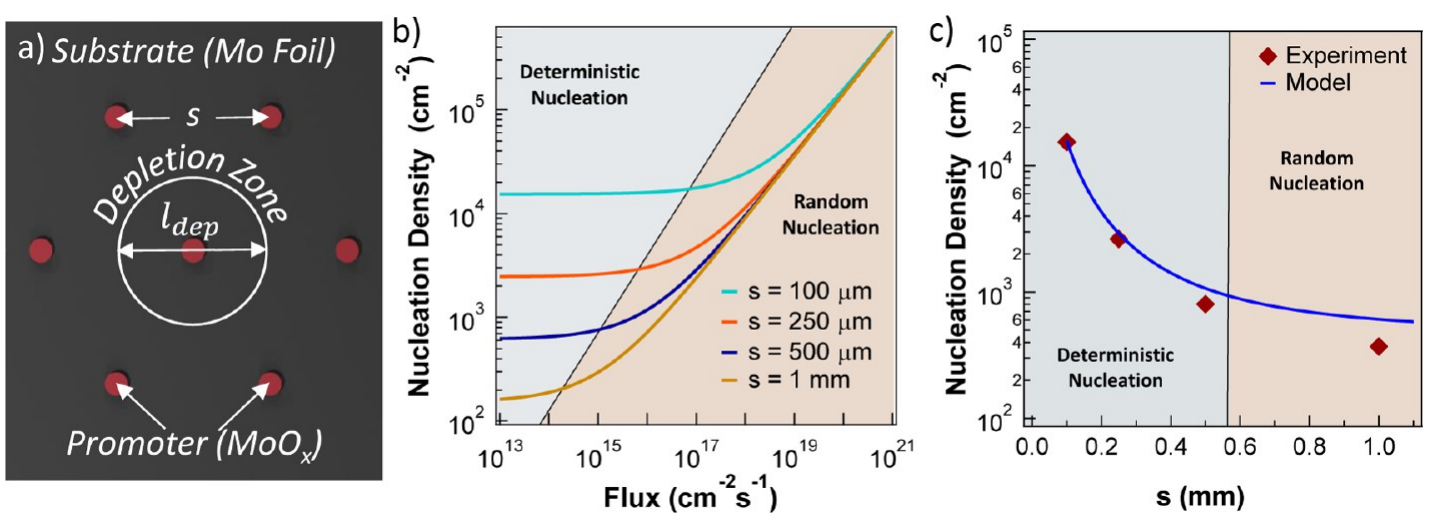

Figure 4. Theory of deterministic nucleation. (a) Schematic of a $\mathrm{Mo} / \mathrm{MoO}_{x}$ patterned substrate with inter nucleation promoter spacing, $s$. Representation of a phosphorus depletion zone with diameter, $l_{\text {dep }}$ is marked on the schematic. When $l_{\text {dep }}>s$, majority of InP crystals nucleate selectively on $\mathrm{MoO}_{x}$ sites and not the bare Mo foil. (b) A plot of calculated nucleation density, $N_{\text {Total }}$ on patterned substrates for different $s$ values. (c) Comparison between experimentally measured and calculated nucleation density as a function of $s$.

$\mathrm{MoO}_{x}$ is $\sim 5 \times 10^{4} \mathrm{~cm}^{-2}$. If we assume hexagonal packing of nuclei, we can define an internuclei length scale, $l=(8 / 3 \sqrt{3} 1 /$ $N)^{0.5} \sim 1.24 / \sqrt{ } N$, which corresponds to $l_{\mathrm{Mo}}=580 \mu \mathrm{m}$ and $l_{\mathrm{MoO}_{x}}=55 \mu \mathrm{m}$ for the bare Mo foil and evaporated $\mathrm{MoO}_{x}$, respectively. These numbers subsequently define the limits of nucleation control possible with bare Mo foils and $\mathrm{Mo} / \mathrm{MoO}_{x}$. While further studies must be conducted to thoroughly understand the difference in nucleation on $\mathrm{MoO}_{x}$ versus Mo surfaces, the experimental results on the nucleation density suggest a lower surface energy for $\mathrm{MoO}_{x}$ as compared to Mo.

Given that the nucleation density on $\mathrm{MoO}_{x}$ is 2 orders of magnitude higher than on Mo foil, we now utilize this disparity to engineer the structure of films on a millimeter scale. First, $\mathrm{MoO}_{x}$ dots of $10 \mu \mathrm{m}$ in diameter and $50 \mathrm{~nm}$ in thickness were patterned in a regular hexagonal geometry on Mo foils, as shown in Figure 3a1. These structures were patterned utilizing a lift-off process. Specifically, Mo foils were coated with photoresist (S1818, $4000 \mathrm{rpm}, 30 \mathrm{~s})$, soft baked at $90{ }^{\circ} \mathrm{C}$ for 90 $\mathrm{s}$, and then exposed and developed. $\mathrm{MoO}_{x}$ was then evaporated on the patterned Mo foil/photoresist substrates using the same conditions as the earlier films. Next, the entire stack was sonicated in acetone at room temperature. Finally, the patterned $\mathrm{Mo} / \mathrm{MoO}_{x}$ foils were soaked in boiling acetone for 1 min before being rinsed in IPA. As $\mathrm{MoO}_{x}$ is etched by $\mathrm{H}_{2} \mathrm{O}$, no water rinses were used after $\mathrm{MoO}_{x}$ evaporation. Different pitches of $s=0.1,0.25,0.5$, and $1 \mathrm{~mm}$ were used for the $\mathrm{MoO}_{x}$ dot pattern. Indium and $\mathrm{SiO}_{x}$ films of $1.5 \mu \mathrm{m}$ and $50 \mathrm{~nm}$ were then evaporated on these heterogeneous $\mathrm{Mo} / \mathrm{MoO}$ substrates. The resulting substrates are illustrated schematically in Figure $3 \mathrm{a} 2$.

Next, the films were partially grown at $525{ }^{\circ} \mathrm{C}$ for $3 \mathrm{~min}$ in an ambient of 25 Torr $10 \% \mathrm{PH}_{3}$. This partial growth condition enables sufficient growth of the InP crystals, such that they are clearly observable. Figure 3a3, shows a $1 \mathrm{~cm} \times 0.5 \mathrm{~cm}$ optical image of a partially grown sample with $0.25 \mathrm{~mm} \mathrm{MoO}_{x}$ dot pitch, demonstrating that this method can produce uniform patterned nucleation on a centimeter scale and is limited only by the growth chamber size used here. Figure $3 b$ shows a set of samples with engineered nucleation position and density of InP crystals with different pitch defined as the spacing between nearest neighbor hexagonal $\mathrm{MoO}_{x}$ dots. By visual inspection, we see that nucleation occurred on nearly all $\mathrm{MoO}_{x}$ sites for the 
various pitches explored here, with $\sim 1-3 \%$ of expected $\mathrm{MoO}_{x}$ sites missing. We believe this occurs due to defects during patterning and liftoff. Furthermore, as the pitch increases the ratio of nucleation on the $\mathrm{MoO}_{x}$ sites only to total nucleation $\left(N_{\mathrm{MoO}_{x}} / N_{\text {Tot }}\right)$ decreases. These observations suggest that (i) the induction time for nucleation on the Mo foil is significantly larger than the induction time for nucleation on the $\mathrm{MoO}_{x}$, allowing nucleation on $\mathrm{MoO}_{x}$ to occur first, and (ii) as $\mathrm{MoO}_{x}$ pitch increases, a transition from deterministic nucleation to random nucleation occurs but only at very large pitch size, approaching $1 \mathrm{~mm}$.

\section{DISCUSSION}

These results may be qualitatively understood by utilizing the concept of the reactant "depletion zone" around growing InP crystals. Specifically, if we assume that the $\mathrm{P}$ concentration gradients in the liquid indium relax to the steady state values quickly compared to the time scale for nucleation of InP crystals, then immediately after formation of an InP nucleus, the region surrounding the nucleus will have significantly reduced phosphorus concentrations, preventing further nucleation of InP crystals. We can approximate the length scale of this zone by $l_{\text {dep }} \sim 1.24 / \sqrt{ } N$ as discussed above. It should be noted that it was previously shown that the nucleation density, and consequently $l_{\text {dep }}$, are a function of the incident $\mathrm{P}$ flux. ${ }^{6}$ Specifically, because InP crystals first nucleate on the $\mathrm{MoO}_{x}$, a depletion zone surrounds each nucleation promoter site (Figure $4 \mathrm{a}$ ). If $l_{\text {dep, }}$, which is set by the $\mathrm{P}$ flux, is larger than the spacing between nucleation promoters, then effectively all random nucleation will be suppressed on the substrate. Thus, an engineered substrate with patterned arrays of nucleation sites enables user control over the density and position of crystals in the TF-VLS growth mode.

To quantitatively explain the results, we developed a simple model for nucleation on the heterogeneous substrates utilizing a kinetic rate equation approach. Specifically, we modified an approach taken previously, ${ }^{6}$ utilizing the assumption that each nucleation promoter site gives rise to a single stable InP nucleus, and that these nucleation events occur before the first nucleation event on the bare Mo foil, allowing us to modify the coupled differential rate equations as follows:

$$
\begin{aligned}
& \frac{\mathrm{d} n}{\mathrm{~d} t}=\frac{F}{h}-\eta D \sigma_{1} n^{\eta}-D \sigma_{\mathrm{s}} n\left(N+N_{0}\right) \\
& \frac{\mathrm{d} N}{\mathrm{~d} t}=D \sigma_{1} n^{\eta}
\end{aligned}
$$

where $F$ is the net flux of $\mathrm{P}$ atoms through the cap $\left(\mathrm{cm}^{-2}-\mathrm{s}^{-1}\right), h$ is the height of the initial In film, $D$ is the diffusivity of $\mathrm{P}$ in liquid In at the process temperature, $\sigma_{1}$ is the capture coefficient for single $\mathrm{P}$ atoms to form an InP nucleus, $\sigma_{\mathrm{s}}$ is the capture coefficient for stable $\mathrm{InP}$ nuclei, $n$ is the excess concentration of $\mathrm{P}$ in the liquid $\mathrm{In}, \eta$ is the number of $\mathrm{P}$ atoms in the critical nucleus, $N$ is the density of stable InP nuclei on the Mo foil, and $N_{0}$ is the density of stable InP nuclei on the $\mathrm{MoO}_{x}$ sites.

It is assumed that (i) the concentration of $\mathrm{P}$ achieves steady state, $\mathrm{d} n / \mathrm{d} t=0$, and (ii) $\eta D \sigma_{1} n^{\eta} \ll D \sigma_{\mathrm{s}} n\left(N+N_{0}\right)$. This is a reasonable assumption given our previous calibration to experimental data that shows $\eta=4$ is the best fit. ${ }^{6}$ Since the critical nucleus size is small and the nucleation density is low, the number of $\mathrm{P}$ atoms captured due to nucleation will be much smaller than the number captured by growing stable crystals.
Using these approximations, it is possible to solve the coupled rate equations to obtain the solution $N=A / h^{2}\left(F h^{4} / D\right)^{\alpha}-N_{0}$, where $\alpha=(\eta-1) /(\eta+1)$, and $A$ is a magnitude fitting parameter. Since $N \geq 0$, the total nucleation density becomes

$$
N_{\text {Total }}=\left\{\begin{array}{cc}
N_{0}, & \frac{A}{h^{2}}\left(\frac{F h^{4}}{D}\right)^{\alpha}-N_{0}<0 \\
\frac{A}{h^{2}}\left(\frac{F h^{4}}{D}\right)^{\alpha}, & \frac{A}{h^{2}}\left(\frac{F h^{4}}{D}\right)^{\alpha}-N_{0} \geq 0
\end{array}\right.
$$

This can be approximated by the smooth function $N_{\text {Total }} \sim A$ / $h^{2}\left(F h^{4} / D\right)^{\alpha}+N_{0}$, since for most incident fluxes either $A$ / $h^{2}\left(F h^{4} / D\right)^{\alpha} \gg N_{0}$ or $A / h^{2}\left(F h^{4} / D\right)^{\alpha} \ll N_{0}$. When the two terms are similar, the maximum error will be a factor of 2 . Furthermore, this equation will be valid when the (i) density of $\mathrm{MoO}_{x}$ nucleation sites, $N_{0}$ is less than the nucleation density on a planar $\mathrm{MoO}_{x}$ film, and (ii) the nucleation on the $\mathrm{MoO}_{x}$ sites occurs before nucleation on the Mo substrate.

The model predicts two regimes: (i) the deterministic nucleation regime, where nucleation occurs entirely on the nucleation promoter sites and (ii) the random nucleation regime, where nucleation occurs on both the promoter sites and the bare substrate. Since $l_{\text {dep }}$ is a function of incident flux, it is expected that the crossover between deterministic and random nucleation occurs at different flux values depending on the pitch of the patterned promoter dots. Figure $4 \mathrm{~b}$ shows a plot of $N_{\text {Total }}$ as a function of incident flux for different promoter pitches using previously calibrated values for A and $\alpha^{6}$. The curves for each pitch show the two nucleation regimes as a function of flux, with the crossover flux decreasing as pitch is increased. Similarly, Figure $4 c$ shows the nucleation density as a function of pitch at a constant flux, a situation which corresponds to the experimental series presented in Figure $3 \mathrm{~b}$. By extracting the flux for the experimental growth conditions, we can plot the expected nucleation density as a function of pitch and compare those values to experiment. The flux is extracted using a procedure previously reported ${ }^{6}$ and found to be $\sim 7 \times 10^{15} \mathrm{~cm}^{-2} \mathrm{~s}^{-1}$. As shown in Figure 4c, both experiment and theory are in good accord, further validating the nucleation mechanism proposed here.

\section{CONCLUSION}

In conclusion, we have demonstrated a simple method to control nuclei position and nucleation density of InP films grown via the TF-VLS growth mode. Critically, we show that it is possible to obtain millimeter scale internuclei spacing and precise control over nucleation position. This corresponds to grain sizes approaching millimeter scale for fully grown thin films on nonepitaxial substrates. The ultralarge grain size is important for the eventual device performance since the grain boundaries that often lead to carrier recombination and/or leakage currents are minimized. Furthermore, we show that a characteristic length, set by the substrate nucleation density, demarcates the boundary between the promoter nucleation and random nucleation regimes and may be used as a design parameter for other material systems and growth conditions. As grain boundaries are often a primary cause of carrier loss in polycrystalline devices, the ability to deterministically position grains in continuous, polycrystalline thin films offers an unprecedented level of control over the nonepitaxially grown film structure. In the future, this method could be leveraged 
such that each device falls on a single millimeter scale grain, enabling a polycrystalline material to appear as a single crystal material from a device perspective.

\section{ASSOCIATED CONTENT}

\section{S Supporting Information}

Material characterization of grown InP. This material is available free of charge via the Internet at http://pubs.acs.org.

\section{AUTHOR INFORMATION}

\section{Corresponding Author}

*E-mail: ajavey@berkeley.edu.

Notes

The authors declare no competing financial interest.

\section{ACKNOWLEDGMENTS}

The experimental part of this work was funded by Bay Area Photovoltaic Consortium. The nucleation modeling and theory was funded by the Director, Office of Science, Office of Basic Energy Sciences, Materials Sciences and Engineering Division of the U.S. Department of Energy under Contract No. DEAC02-05CH11231.

\section{REFERENCES}

(1) Green, M. A. Prog. Photovoltaics 2012, 20 (4), 472-476.

(2) Green, M. A.; Emery, K.; Hishikawa, Y.; Warta, W.; Dunlop, E. D. Prog. Photovoltaics 2013, 21 (5), 827-837.

(3) Miller, O. D.; Yablonovitch, E.; Kurtz, S. R. IEEE J. Photovoltaics 2012, 2 (3), 303-311.

(4) Kayes, B. M.; Nie, H.; Twist, R.; Spruytte, S. G.; Reinhardt, F.; Kizilyalli, I. C.; Higashi, G. S. 27.6\% conversion efficiency, a new record for single-junction solar cells under 1 sun illumination. 37th IEEE Photovoltaic Specialists Conference (PVSC), IEEE: New York, 2011; pp 4-8.

(5) Keavney, C.; Haven, V.; Vernon, S. Emitter structures in MOCVD InP solar cells. Conference Record of the 21st IEEE Photovoltaic Specialists Conference, IEEE: New York, 1990; pp 141144.

(6) Kapadia, R.; Yu, Z.; Wang, H.-H. H.; Zheng, M.; Battaglia, C.; Hettick, M.; Kiriya, D.; Takei, K.; Lobaccaro, P.; Beeman, J. W. Sci. Rep. 2013, 3, 2275.

(7) Wagner, R.; Ellis, W. Appl. Phys. Lett. 1964, 4 (5), 89-90.

(8) Morales, A. M.; Lieber, C. M. Science 1998, 279 (5348), 208211.

(9) Pan, Z. W.; Wang, Z. L. Science 2001, 291 (5510), 1947-1949.

(10) Fan, Z.; Razavi, H.; Do, J.-w.; Moriwaki, A.; Ergen, O.; Chueh, Y.-L.; Leu, P. W.; Ho, J. C.; Takahashi, T.; Reichertz, L. A. Nat. Mater. 2009, 8 (8), 648-653.

(11) Ergen, O.; Ruebusch, D. J.; Fang, H.; Rathore, A. A.; Kapadia, R.; Fan, Z.; Takei, K.; Jamshidi, A.; Wu, M.; Javey, A. J. Am. Chem. Soc. 2010, 132, 13972.

(12) Britt, J.; Ferekides, C. Appl. Phys. Lett. 1993, 62, 2851.

(13) Kiriya, D.; Zheng, M.; Kapadia, R.; Zhang, J.; Hettick, M.; Yu, Z.; Takei, K.; Hank Wang, H.-H.; Lobaccaro, P.; Javey, A. J. Appl. Phys. 2012, 112 (12), 123102-123102-6.

(14) Zheng, M.; Yu, Z.; Joon Seok, T.; Chen, Y.-Z.; Kapadia, R.; Takei, K.; Aloni, S.; Ager, J. W.; Wu, M.; Chueh, Y.-L. J. Appl. Phys. 2012, 111 (12), 123112-123112-6.

(15) Kurtz, S. R.; McConnell, R. AIP Conf. Proc. 1997, 191.

(16) Yamaguchi, M.; Itoh, Y. J. Appl. Phys. 1986, 60 (1), 413-417.

(17) Kapadia, R.; Fan, Z.; Javey, A. Appl. Phys. Lett. 2010, 96, 103116. 
Deterministic nucleation of InP on metal foils with the thin-film vapor-liquidsolid growth mode

Rehan Kapadia ${ }^{1,2}$, Zhibin $\mathrm{Yu}^{1,2}$, Mark Hettick ${ }^{1,2}$, Jingsan $\mathrm{Xu}^{1,2}$, Maxwell S. Zheng ${ }^{1,2}$, ChengYing Chen ${ }^{1,2}$, Arunima D. Balan ${ }^{1,2}$, Daryl C. Chrzan ${ }^{1,2}$, Ali Javey ${ }^{1,2^{*}}$

${ }^{I}$ Electrical Engineering and Computer Sciences, University of California, Berkeley, CA 94720

${ }^{2}$ Materials Sciences Division, Lawrence Berkeley National Laboratory, Berkeley, CA 94720

*ajavey@berkeley.edu

\section{Supporting Information}



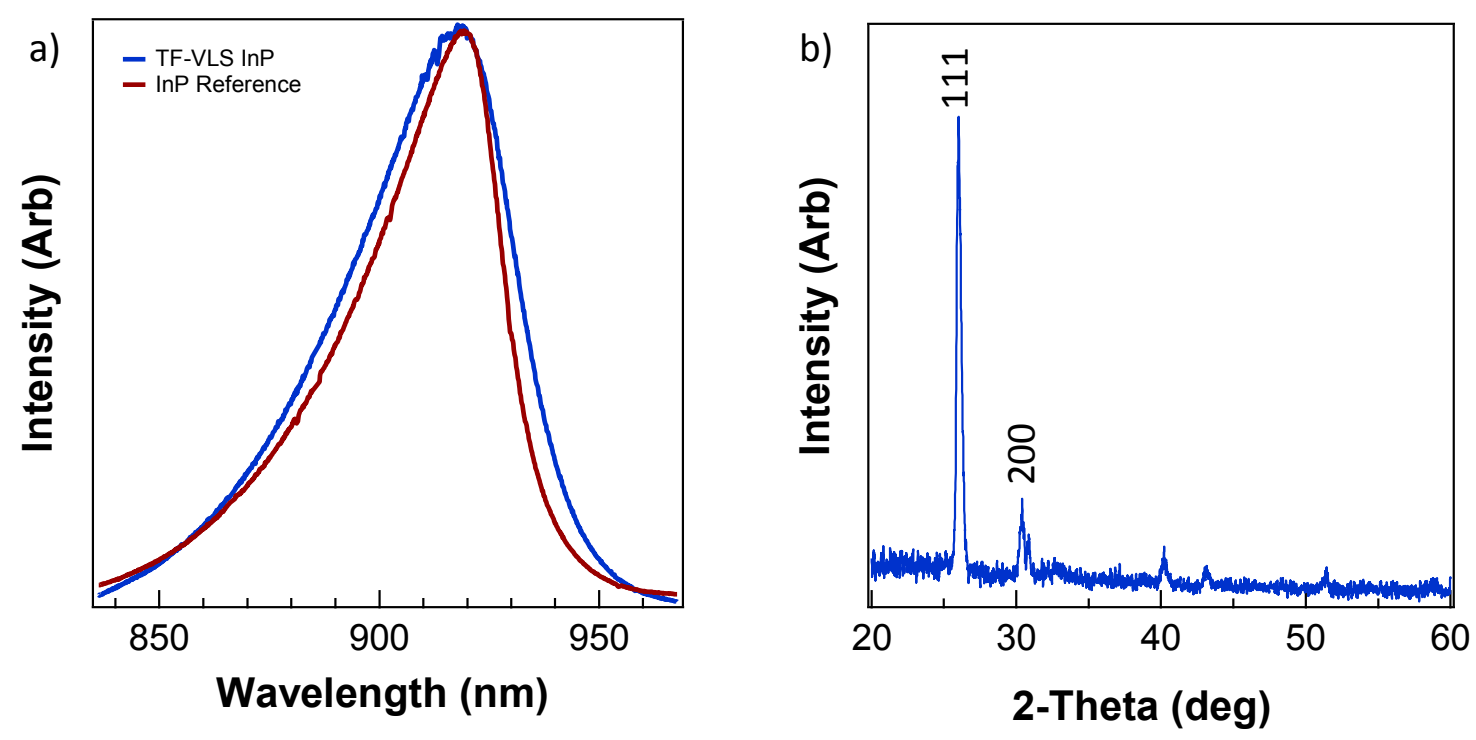

Figure S1: Material characterization of the grown InP. (a) steady state photoluminescence spectra and (b) x-ray diffraction patterns taken from the grown InP islands. The prominent InP peaks $(111,200)$ are labeled in (b). 